\title{
Baseline LDL-C and Lp(a) Elevations Portend a High Risk of Coronary Revascularization in Patients after Stent Placement
}

\author{
Anping Cai, ${ }^{1}$ Liwen Li, ${ }^{1}$ Ying Zhang, ${ }^{1}$ Yujin Mo, ${ }^{1}$ Zhigen Li, \\ Weiyi Mai, ${ }^{2}$ and Yingling Zhou ${ }^{1}$ \\ ${ }^{1}$ Department of Cardiology, Guangdong Cardiovascular Institute, Guangdong General Hospital, \\ Guangdong Academy of Medical Sciences, 106 Zhongshan Road 2, Guangzhou 510080, China \\ ${ }^{2}$ Department of Cardiology, The First Affiliated Hospital of Sun Yat-sen University, Guangzhou 510080, China
}

Correspondence should be addressed to Yingling Zhou; drcacocolacai@gmail.com

Received 14 August 2013; Revised 29 October 2013; Accepted 3 November 2013

Academic Editor: Olav Lapaire

Copyright (C) 2013 Anping Cai et al. This is an open access article distributed under the Creative Commons Attribution License, which permits unrestricted use, distribution, and reproduction in any medium, provided the original work is properly cited.

\begin{abstract}
Background and Aim. Incidence of coronary restenosis after stent placement is high. Our study was going to investigate whether $\mathrm{Lp}$ (a) elevation was potential for predicting coronary restenosis and whether the effects of Lp(a) elevation on coronary restenosis were dependent on LDL-C level. Methods and Results. Totally 832 participants eligible for stent placement were enrolled and followed up for monitoring clinical end points. Baseline characteristics were collected. According to the cut point of Lp(a), participants were divided into low $\mathrm{Lp}(\mathrm{a})$ group $(\mathrm{Lp}(\mathrm{a})<30 \mathrm{mg} / \mathrm{dL})$ and high $\mathrm{Lp}(\mathrm{a})$ group $(\mathrm{Lp}(\mathrm{a}) \geq 30 \mathrm{mg} / \mathrm{dL})$. Furthermore, based on baseline LDL-C level, participants were divided into LDL-C $<1.8 \mathrm{mmol} / \mathrm{L}$ and $\geq 1.8 \mathrm{mmol} / \mathrm{L}$ subgroups. Clinical end points including major adverse cardiovascular events (MACE), cardiovascular death, nonfatal myocardial infarction, ischemic stroke, and coronary revascularization $(\mathrm{CR})$ were compared. Patients in high $\mathrm{Lp}(\mathrm{a})$ groups more frequently presented with acute coronary syndrome and three vessel stenoses. In subgroup of LDL-C $<1.8 \mathrm{mmol} / \mathrm{L}$, no significant differences of cardiovascular outcomes were found between low and high Lp(a) groups. While in the subgroup of LDL-C $\geq 1.8 \mathrm{mmol} / \mathrm{L}$, incidences of MACE and CR were significantly higher in high Lp(a) group, and odds ratio for CR was 2.05. Conclusion. With baseline LDL-C and Lp(a) elevations, incidence of $\mathrm{CR}$ is significantly increased after stent placement.
\end{abstract}

\section{Introduction}

Dyslipidemias, especially characterized by high serum level of low density lipoprotein-cholesterol (LDL-C), has been well documented as an important risk factor of atherosclerosis and its manifestation of atherosclerotic cardiovascular diseases (CVD) [1]. In the past decades, accumulating evidence from clinical studies firmly demonstrates that LDL-C decreased by lipid-lowering drug, such as HMG-CoA reductase inhibitor (statins), is crucial for reducing the incidence of coronary heart diseases (CHD), ischemic stroke, and peripheral artery diseases [2-5]. However, some epidemiological studies show that, although target LDL-C level has been achieved by statins therapy, residual cardiovascular risk such as restenosis after stent placement in some population groups is still very high [6-8], suggesting that, besides LDL-C, other risk factors could also play a contributory role in the progression and recurrence of CVD, and identifying those potential risk factors would be useful, helpful, and beneficial for further improving cardiovascular outcome.

Lipoprotein(a) (Lp(a)), composed of an LDL-C enriched core with one molecular of apolipoprotein B-100 (apoB100) and apolipoprotein(a) (apo(a)) via a disulfide bond, is currently considered a potential important candidate as supported by previous epidemiological, animal, and genetic epidemiological studies [9-12]. In comparison with LDL-C, $\mathrm{Lp}$ (a) not only is capable of promoting atherosclerosis, but also has robust effects on enhancing thrombus formation by means of impairing fibrinolysis $[13,14]$. Therefore, Lp(a) elevation is believed to be the very likely risk factor accounting for residual cardiovascular risk, especially in patients with target LDL-C level. Nevertheless, findings from previous reported studies and meta-analysis are not consistent [15-19]. For example, some epidemiological studies show that the risk 
$\mathrm{Lp}$ (a) imposes on cardiovascular system is overlapped with LDL-C [16-18], whereas other studies reveal a quite opposite outcome in which $\operatorname{Lp}(\mathrm{a})$ is an independent predictor for cardiovascular risk and traditional risk factor such as LDLC plays no role in risk estimation for $\operatorname{Lp}(\mathrm{a})$ elevation $[15,19]$. In retrospect, these discrepancies regarding the predictive value of $\mathrm{Lp}(\mathrm{a})$ elevation on cardiovascular outcomes may be partially associated with the level of LDL-C which warrants further investigation.

Nowadays, percutaneous coronary intervention (PCI) is the preferred choice for revascularization worldwide. However, it is noted that a large number of patients after stent placement, in spite of achieving recommended LDL-C level with statins therapy, have target vessel restenoses which require revascularization. Therefore, preventing and reducing the incidence of coronary restenosis after stent placement is of paramount importance. Notably, the pathophysiological process of coronary artery restenosis is featured by smooth muscle cells' overproliferation, endothelial dysfunction, and fibrin accumulation, and with respect to $\operatorname{Lp}(\mathrm{a})$ unique features as to proatherosclerosis and prothrombosis, we speculated that $\operatorname{Lp}(\mathrm{a})$ elevation might be the potential factor responsible for increased incidence of cardiovascular events, particularly for coronary artery restenosis in patients after stent placement. Furthermore, whether the effects of $\operatorname{Lp}(\mathrm{a})$ elevation on cardiovascular events' recurrence after stent placement are dependent on LDL-C level or not would also be investigated.

\section{Method}

2.1. Study Population and Protocol. Our current study has been registered in the Chinese Clinical Trial Registry (Registration number: ChiCTR-OCH-11001198). Written informed consent was obtained and the ethics committee of the Guangdong General Hospital approved present study. All participants were enrolled from Guangdong General Hospital.

After coronary artery angiography, participants eligible for coronary artery stent placement were enrolled. Demographic and clinical characteristics of all participants were collected at the initial clinical contact (see Table 1), and all participants were followed up after stent placement via outpatient visit or telephone call for monitoring event occurrence for totally 1 year. Accordingly [1], patient with poststent placement is recommended to achieve target LDL-C level of less than $1.8 \mathrm{mmol} / \mathrm{L}$; therefore, we used $1.8 \mathrm{mmol} / \mathrm{L}$ of LDL-C as cut-off point to categorize all patients into two subgroups.

2.2. Laboratory Measurement. Blood samples were drawn by venipuncture in the morning when participants were fasting for at least 8 hours for the variables measurement. Of note, plasma level of $\operatorname{Lp}(\mathrm{a})$ was measured with sandwich enzymelinked immune-sorbent assays (ELISA kit, Yaji Biosystems, Shanghai, China). All the procedures were performed in accordance with the manual instructions and were evaluated by SYNCHRON LX20 UniCel DxC800 analyzer (Beckman Coulter Inc., USA). Accordingly [9], plasma level of Lp(a) lower than $30 \mathrm{mg} / \mathrm{dL}$ is considered within the normal range and equal or higher than $30 \mathrm{mg} / \mathrm{dL}$ is recognized as abnormal.

2.3. Clinical End Points Definition and Assessment. In our current study, clinical end points after stent placement were defined as cardiovascular death, nonfatal myocardial infarction, nonfatal ischemic stroke, and coronary revascularization. Assessment of the incidence of clinical end points after stent placement was performed during followup by means of outpatient visit and/or telephone call after discharge.

2.4. Statistical Analysis. Continuous data was presented as mean \pm SD or median (interquartile range) appropriately and compared by Student's $t$-test when data was normally distributed, otherwise compared by the Wilcoxon ranksum test. Categorical data was presented as percentage and compared by $\chi^{2}$ test. Statistical analyses were performed by using SPSS software version 16.0 (SPSS, Inc., Chicago, Illinois). A value of $P<0.05$ was considered significant.

\section{Results}

3.1. Baseline Characteristics of Low and High Lp(a) Groups. As shown in Table 1, the percentages of patients presented with acute coronary syndrome and three vessel stenoses were significantly higher in high $\mathrm{Lp}(\mathrm{a})$ group than those in low $\mathrm{Lp}(\mathrm{a})$ groups, indicating that patients with $\operatorname{Lp}(\mathrm{a})$ elevation were more prone to have more critical coronary artery stenoses. And the percentage of left main coronary artery lesion in high Lp(a) group was higher than that in the low Lp(a) group though without insignificant difference $(14.3 \%$ versus $11.8 \%$, $P=0.321)$. Other baseline characteristics between these two groups were comparable. All participants enrolled in our study were well followed up and were strictly adhering to recommended medications such as dual antiplatelet and statins therapies.

3.2. Cardiovascular Outcomes Comparison in the Subgroup of LDL-C Lower Than $1.8 \mathrm{mmol} / \mathrm{L}$ after Stent Placement. Selecting LDL-C level of $1.8 \mathrm{mmol} / \mathrm{L}$ as the cut-off point, 823 participants were divided into two groups. In the subgroup of LDL-C level lower than $1.8 \mathrm{mmol} / \mathrm{L}$, there were 287 patients with $\mathrm{Lp}$ (a) level lower than $30 \mathrm{mg} / \mathrm{dL}$, while another 92 were with Lp(a) level higher than $30 \mathrm{mg} / \mathrm{dL}$. As shown in Table 2, no significant differences of MACE were found between low and high $\mathrm{Lp}(\mathrm{a})$ groups $(16.3 \%$ versus $18.5 \%, P=0.755)$. And each individual end point between these two groups was also comparable. Notably, among each individual outcome, the incidence of coronary revascularization was higher than the others in both groups.

3.3. Cardiovascular Outcomes Comparison in the Subgroup of LDL-C Equal or Higher Than $1.8 \mathrm{mmol} / \mathrm{L}$ after Stent Placement. Another 383 patients with LDL-C level equal or higher than $1.8 \mathrm{mmol} / \mathrm{L}$ were divided into two groups according to the cut-off point of $\operatorname{Lp}(\mathrm{a})$. As shown in Table 2, there was significant difference of incident MACE after stent placement between high and low Lp(a) groups (26.1\% versus $16.6 \%$, 
TABLE 1: Comparison of baseline characteristics of low-Lp(a) and high-Lp(a) groups.

\begin{tabular}{|c|c|c|c|}
\hline Variables & Low-Lp(a) $(n=552)$ & High-Lp(a) $(n=280)$ & $P$ value \\
\hline Age (years) & $62.2 \pm 10.3$ & $62.3 \pm 11.1$ & 0.107 \\
\hline Male (\%) & 83.9 & 87.5 & 0.181 \\
\hline Smoking (\%) & 48.6 & 43.2 & 0.162 \\
\hline $\mathrm{SBP}(\mathrm{mmHg})$ & $130.4 \pm 20.5$ & $126.3 \pm 17.8$ & 0.089 \\
\hline $\mathrm{DBP}(\mathrm{mmHg})$ & $75.8 \pm 11.3$ & $74.5 \pm 10.0$ & 0.192 \\
\hline HTN (\%) & 56.9 & 51.1 & 0.122 \\
\hline GLU (mmol/L) & $5.84 \pm 1.67$ & $5.80 \pm 1.82$ & 0.920 \\
\hline $\mathrm{GHBA}_{1} \mathrm{C}(\%)$ & $6.31 \pm 1.02$ & $6.31 \pm 1.17$ & 0.968 \\
\hline DM (\%) & 24.6 & 20.0 & 0.140 \\
\hline $\mathrm{CHOL}(\mathrm{mmol} / \mathrm{L})$ & $4.05 \pm 1.13$ & $4.31 \pm 1.19$ & 0.406 \\
\hline LDL-C (mmol/L) & $2.33 \pm 0.88$ & $2.64 \pm 0.89$ & 0.184 \\
\hline HDL-C (mmol/L) & $1.04 \pm 0.31$ & $1.04 \pm 0.30$ & 0.500 \\
\hline $\log \mathrm{TG}(\mathrm{mmol} / \mathrm{L})$ & $0.16 \pm 0.25$ & $0.11 \pm 0.20$ & 0.001 \\
\hline APOA (mmol/L) & $1.01 \pm 0.23$ & $0.97 \pm 0.24$ & 0.465 \\
\hline $\mathrm{APOB}(\mathrm{mmol} / \mathrm{L})$ & $0.67 \pm 0.17$ & $0.75 \pm 0.18$ & 0.364 \\
\hline CREA (umol/L) & $97.3 \pm 31.9$ & $96.2 \pm 34.4$ & 0.984 \\
\hline Uric acid (umol/L) & $366.1 \pm 154.8$ & $380.0 \pm 101.6$ & 0.893 \\
\hline $\operatorname{LVEF}(\%)$ & $60.65 \pm 10.45$ & $59.58 \pm 10.77$ & 0.534 \\
\hline ACS (\%) & 67.0 & 78.2 & 0.001 \\
\hline LM (\%) & 11.8 & 14.3 & 0.321 \\
\hline Trivessel (\%) & 35.5 & 48.2 & 0.001 \\
\hline Previous PCI (\%) & 2.5 & 4.3 & 0.205 \\
\hline Previous CABG (\%) & 1.4 & 1.1 & 0.759 \\
\hline Previous stroke (\%) & 4.5 & 4.3 & 1.000 \\
\hline Aspirin (\%) & 100 & 100 & \\
\hline Clopidogrel (\%) & 100 & 100 & \\
\hline Statins (\%) & 100 & 100 & \\
\hline
\end{tabular}

HTN: hypertension, GLU: glucose (fasting), $\mathrm{HA}_{1} \mathrm{C}$ : glycated hemoglobin, DM: diabetes mellitus, CHOL: total cholesterol, TG: triglyceride, CREA: creatinine, LVEF: left ventricular ejection fraction, ACS: acute coronary syndrome, LM: left main.

TABLE 2: Cardiovascular outcomes comparison in the different LDL-C subgroup after stent placement.

\begin{tabular}{|c|c|c|c|}
\hline Variables & Low-Lp(a) $(n=287)$ & High-Lp(a) $(n=92)$ & $P$ value \\
\hline \multicolumn{4}{|c|}{ LDL-C $<1.8$ subgroup $(n=379)$} \\
\hline MACE (\%) & $53(18.5)$ & $15(16.3)$ & 0.755 \\
\hline Death (\%) & $6(2.1)$ & $2(2.2)$ & 0.617 \\
\hline MI (\%) & $4(1.4)$ & $2(2.2)$ & 0.449 \\
\hline Stroke $(\%)$ & $5(1.7)$ & $3(3.3)$ & 0.302 \\
\hline CR (\%) & $38(13.2)$ & $8(8.7)$ & 0.164 \\
\hline Variables & Low-Lp(a) $(n=265)$ & High-Lp(a) $(n=118)$ & $P$ value \\
\hline \multicolumn{4}{|c|}{ LDL-C $>1.8$ subgroup $(n=453)$} \\
\hline MACE (\%) & $44(16.6)$ & $49(26.1)$ & 0.018 \\
\hline Death $(\%)$ & $15(5.7)$ & $10(5.3)$ & 0.525 \\
\hline MI (\%) & $6(2.3)$ & $5(2.7)$ & 0.509 \\
\hline Stroke $(\%)$ & $3(1.1)$ & $5(2.7)$ & 0.196 \\
\hline $\mathrm{CR}(\%)$ & $20(7.5)$ & $29(15.4)$ & 0.006 \\
\hline
\end{tabular}

MACE: major adverse cardiovascular event, MI: myocardial infarction (nonfatal), CR: coronary revascularization. 
$P=0.018$ ), and this difference was predominantly derived from the higher incidence of coronary revascularization in high $\mathrm{Lp}$ (a) group $(15.4 \%$ versus $7.5 \%, P=0.006)$, and the odds ratio for coronary revascularization in high $\mathrm{Lp}$ (a) group was 2.05, whereas the incidence of the other individual end point was similar between groups. Similar to that with LDL-C lower than $1.8 \mathrm{mmol} / \mathrm{L}$, the percentage of coronary revascularization in low and high $\operatorname{Lp}(\mathrm{a})$ groups was still higher than other clinical end points, indicating that in poststent placement, target vessel restenosis was the most significant residual cardiovascular risk, regardless of LDL-C level.

\section{Discussion}

Our study totally enrolled 832 patients diagnosed with coronary artery disease who were eligible for stent placement after coronary angiography examination, and the results showed that (1) after stent placement, rather than an independent predictor, the predictive value of $L p(a)$ elevation on cardiovascular outcomes, especially coronary revascularization, was dependent on baseline LDL-C level; (2) in the subgroup of baseline LDL-C $\geq 1.8 \mathrm{mmol} / \mathrm{L}$, patients with high Lp(a) level have increased incidence of MACE than patients with low Lp(a) level, and the between-group difference was mainly derived from coronary revascularization. Nevertheless, in the subgroup of baseline LDL-C $<1.8 \mathrm{mmol} / \mathrm{L}$, the incidence of MACE after stent placement was comparable between high and low Lp(a) level groups; (3) moreover, in comparison to patients with low $\operatorname{Lp}(\mathrm{a})$ level, patients with high Lp(a) level more often presented with acute coronary syndrome and three vessel stenoses.

Currently, percutaneous coronary intervention is broadly applied in patients with significant coronary artery stenosis, and this reperfusion strategy has profoundly improved cardiovascular outcomes in the past two decades. However, accumulating evidence from retrospective and perspective studies shows that, despite stent placement and strictly adhering to contemporary guideline recommended medications, a substantial number of patients still have a high residual cardiovascular risk, especially target vessel restenoses which necessitate revascularization. With respect to these findings, many strategies aiming to reduce residual cardiovascular risk have been introduced, and more aggressive LDL-C lowering is considered as one of the most attractive and promising strategies. In the PROVE IT-TIMI 22 trial [6], Cannon CP and colleagues observed that in patients with acute coronary syndrome, intensive therapy ( $80 \mathrm{mg}$ of atorvastatin daily) conferred greater benefits of mortality and MACE than those of standard therapy ( $40 \mathrm{mg}$ of pravastatin daily), which was believed attributed to further reduction of LDL-C by intensive lipid lowering therapy (LDL-C $1.60 \mathrm{mmol} / \mathrm{L}$ versus $2.46 \mathrm{mmol} / \mathrm{L}, P<0.001)$. However, after nearly 2 years' followup, the incidence of revascularization in intensive therapy group was still up to $16.3 \%$. Additionally, in the IDEAL and TNT trials, after approximately 5 years' followup, $12.0 \%$ and $8.7 \%$ of MACE were observed, respectively, $[7,8]$. Findings of these large clinical trials consistently show that significant residual cardiovascular risks still remain in patient despise with aggressive LDL-C reduction, and LDL-C alone is inadequate to fully evaluate and predict the residual cardiovascular risk. In lieu of evidence from epidemiological studies [20-22], Lp(a) elevation has been recognized as a potential candidate at least partially responsible for high residual cardiovascular risk in patient achieving target LDL-C level. However, interaction between Lp(a) and LDL-C on cardiovascular outcome has been observed and whether the effects of $\mathrm{Lp}(\mathrm{a})$ elevation on cardiovascular outcome are independent of or dependent on LDL-C is still unclear.

Our current study showed that the predictive value of $\mathrm{Lp}(\mathrm{a})$ elevation for MACE, exclusively for coronary revascularization, in CHD patients with stent placement is predominantly dependent on baseline level of LDL-C. In the subgroup of baseline LDL-C level $\geq 1.8 \mathrm{mmol} / \mathrm{L}$, the incidence of revascularization for patients in high $\mathrm{Lp}(\mathrm{a})$ group was significantly increased and the odds ratio was 2.05 when compared to low level group (15.4\% versus $7.5 \%$, $P=0.006)$, whereas there was no significant difference of other individual outcome (see Table 2). Furthermore, in the subgroup of LDL-C $<1.8 \mathrm{mmol} / \mathrm{L}$, all cardiovascular outcomes between low and high $\mathrm{Lp}(\mathrm{a})$ groups were insignificantly different (see Table 2). The unique features of $\mathrm{Lp}(\mathrm{a})$ may be partially responsible for these findings. Accordingly, the key component of $\mathrm{Lp}(\mathrm{a})$ in terms of LDL-C enriched core abounds with oxidized phospholipid (OxPL) which is a potent proinflammatory and prooxidative compound that can significantly enhance the pro-atherosclerotic potential of $\operatorname{Lp}(\mathrm{a})$ [23], while in patients with extreme low level of LDL-C (less than $1.8 \mathrm{mmol} / \mathrm{L}$ accordingly), the risk of $\mathrm{Lp}(\mathrm{a})$ elevation on cardiovascular system is diminished or even disappeared, and the underlying mechanisms are not fully understood yet. To our best knowledge, there may be two aspects attributed. In the first place, it was reported that $\mathrm{Lp}(\mathrm{a})$ degradation is partially mediated by LDL-C receptor, although the role by this pathway is not fully conclusive yet [24]. Therefore, higher LDL-C level may compete for LDL-C receptor which in turn reduces Lp(a) catabolism and amplifies Lp(a) biological effects, and this may be one of the underlying mechanisms attributed to the synergistic effects of both LDL-C and Lp(a) elevations, while in very low LDL-C level $(<1.8 \mathrm{mmol} / \mathrm{L})$, this synergistic effect between LDL-C and $\operatorname{Lp}(\mathrm{a})$ may be waned and unappreciated as indicated by our current study and previous studies [18, 21]. Secondly, it has been identified that, in the normolipidemic individuals, $\mathrm{Lp}(\mathrm{a})$ is capable of removing oxidized phospholipids (such as oxLDL-C) from circulation system via its ability to biding these proinflammatory and prothrombosis materials [23]. Therefore, with the higher level of LDL-C, increased Lp(a) level has higher risk of cardiovascular events than that with lower Lp(a) level, while in the lower level of LDL-C, presumed lower oxLDL-C level, Lp(a) elevation may be beneficial both for oxLDL-C clearance and cardiovascular outcomes, as supported by our current study that, in the subgroup of LDL-C lower than $1.8 \mathrm{mmol} / \mathrm{L}$, the incidence of MACE was slightly lower in high $\mathrm{Lp}(\mathrm{a})$ group than that in low $\mathrm{Lp}(\mathrm{a})$ group $(16.3 \%$ versus $18.5 \%, P=0.755)$, though insignificantly. 
Finally, our current study shows that, in the subgroup of high LDL-C level, the incidence of coronary revascularization after stent placement is positively associated with $\mathrm{Lp}(\mathrm{a})$ level. Accordingly, $\mathrm{Lp}(\mathrm{a})$ is capable of impairing tissue growth factor beta (TGF- $\beta$ ) activity which results in smooth muscle cells' proliferation and migration [25]. Furthermore, the results from Bruneck study support the notion that fibrinolysis attenuation by $\operatorname{Lp}(\mathrm{a})$ is crucial for stabilizing atheroma-attached fibrin thrombi [26]. Lastly, Lp(a) elevation promotes endothelial dysfunction which also contributes to the restenosis progress [27]. The higher percentage of patients presented with acute coronary syndrome and three vessel stenoses also indicates that the thrombi burden in high $\mathrm{Lp}(\mathrm{a})$ groups is greater than that in low $\mathrm{Lp}$ (a) group. Taken together, it is reasonable to make a conclusion that in patients with stent placement, high Lp(a) level may portend a high restenosis rate.

In conclusion, our current study reveals that, in patients with stent placement, $\mathrm{Lp}(\mathrm{a})$ elevation is positively associated with coronary restenosis which requires revascularization. In patients with baseline level of LDL-C $\geq 1.8 \mathrm{mmol} / \mathrm{L}$, the incidence of MACE and coronary revascularization is significantly higher in high Lp(a) group than low Lp(a) group, indicating that, after stent placement, in patient with both baseline levels of LDL-C and Lp(a) elevation, more aggressive LDL-C reduction may mitigate the adverse effects imposed by Lp(a) elevation.

\section{Conflict of Interests}

All authors declare that there is no conflict of interests.

\section{Acknowledgments}

This work was supported by the Grants from the Technology Project Foundation of Guangdong Province, China (2009A030301004, 2011B031800021, 2011B061300034, and 2011B031800263), Guangdong Natural Science Foundation (S2012010009532), Guangdong Medical Research Foundation (B2011002), Cardiovascular Medication Grant of Guangdong Province (2011X25), and Medical Scientific Research Grant of the Health Ministry of Guangdong Province, China (B2011310 and A2012663). Anping Cai and Liwen Li are cofirst authors.

\section{References}

[1] Z. Reiner, A. L. Catapano, G. De Backer et al., "ESC/EAS guidelines for the management of dyslipidaemias: the task force for the management of dyslipidaemias of the European Society of Cardiology (ESC) and the European Atherosclerosis Society (EAS)," European Heart Journal, vol. 32, no. 14, pp. 1769-1818, 2011.

[2] C. M. Gibson, Y. B. Pride, C. P. Hochberg, S. Sloan, M. S. Sabatine, and C. P. Cannon, "Effect of intensive statin therapy on clinical outcomes among patients undergoing percutaneous coronary intervention for acute coronary syndrome. PCIPROVE IT: A PROVE IT-TIMI 22 (Pravastatin or Atorvastatin Evaluation and Infection Therapy-Thrombolysis In Myocardial
Infarction 22) Substudy," Journal of the American College of Cardiology, vol. 54, no. 24, pp. 2290-2295, 2009.

[3] J. Armitage, L. Bowman, K. Wallendszus et al., "Intensive lowering of LDL cholesterol with $80 \mathrm{mg}$ versus $20 \mathrm{mg}$ simvastatin daily in 12,064 survivors of myocardial infarction: a doubleblind randomised trial," The Lancet, vol. 376, no. 9753, pp. 1658$1669,2010$.

[4] C. Baigent, L. Blackwell, J. Emberson et al., "Efficacy and safety of more intensive lowering of LDL cholesterol: a meta-analysis of data from 170,000 participants in 26 randomised trials," The Lancet, vol. 376, no. 9753, pp. 1670-1681, 2010.

[5] T. Ohira, P. J. Schreiner, J. D. Morrisett, L. E. Chambless, W. D. Rosamond, and A. R. Folsom, "Lipoprotein(a) and incident ischemic stroke: The Atherosclerosis Risk in Communities (ARIC) study," Stroke, vol. 37, no. 6, pp. 1407-1412, 2006.

[6] C. P. Cannon, E. Braunwald, C. H. McCabe et al., "Intensive versus moderate lipid lowering with statins after acute coronary syndromes," The New England Journal of Medicine, vol. 350, no. 15, pp. 1495-1504, 2004.

[7] J. C. LaRosa, S. M. Grundy, D. D. Waters et al., "Intensive lipid lowering with atorvastatin in patients with stable coronary disease," The New England Journal of Medicine, vol. 352, no. 14, pp. 1425-1435, 2005.

[8] T. R. Pedersen, O. Faergeman, J. J. P. Kastelein et al., "High-dose atorvastatin vs usual-dose simvastatin for secondary prevention after myocardial infarction: The IDEAL study: a randomized controlled trial," Journal of the American Medical Association, vol. 294, no. 19, pp. 2437-2445, 2005.

[9] B. G. Nordestgaard, M. J. Chapman, K. Ray et al., "Lipoprotein(a) as a cardiovascular risk factor: current status," European Heart Journal, vol. 31, no. 23, pp. 2844-2853, 2010.

[10] S. Tsimikas and J. L. Hall, "Lipoprotein(a) as a potential causal genetic risk factor of cardiovascular disease: a rationale for increased efforts to understand its pathophysiology and develop targeted therapies," Journal of the American College of Cardiology, vol. 60, no. 8, pp. 716-721, 2012.

[11] R. Clarke, J. F. Peden, J. C. Hopewell et al., "Genetic variants associated with $\mathrm{Lp}(\mathrm{a})$ lipoprotein level and coronary disease," The New England Journal of Medicine, vol. 361, no. 26, pp. 2518$2528,2009$.

[12] A. Cai, W. Li, Y. Zhang, Y. Mo, W. Mai, and Y. Zhou, "Lipoprotein(a): a promising marker for residual cardiovascular risk assessment," Disease Markers, vol. 35, no. 5, pp. 551-559, 2013.

[13] L. Berglund and R. Ramakrishnan, "Lipoprotein(a): an elusive cardiovascular risk factor," Arteriosclerosis, Thrombosis, and Vascular Biology, vol. 24, no. 12, pp. 2219-2226, 2004.

[14] P. R. Kamstrup, "Lipoprotein(a) and ischemic heart disease-A causal association? A review," Atherosclerosis, vol. 211, no. 1, pp. 15-23, 2010.

[15] P. R. Kamstrup, M. Benn, A. Tybjærg-Hansen, and B. G. Nordestgaard, "Extreme lipoprotein(a) levels and risk of myocardial infarction in the general population: The Copenhagen City Heart Study," Circulation, vol. 117, no. 2, pp. 176-184, 2008.

[16] G. Luc, J.-M. Bard, D. Arveiler et al., "Lipoprotein (a) as a predictor of coronary heart disease: The PRIME Study," Atherosclerosis, vol. 163, no. 2, pp. 377-384, 2002.

[17] J. S. Danik, N. Rifai, J. E. Buring, and P. M. Ridker, "Lipoprotein(a), measured with an assay independent of apolipoprotein(a) isoform size, and risk of future cardiovascular events among initially healthy women," Journal of the American Medical Association, vol. 296, no. 11, pp. 1363-1370, 2006. 
[18] B. Cantin, F. Gagnon, S. Moorjani et al., "Is lipoprotein(a) an independent risk factor for ischemic heart disease in men? The Quebec cardiovascular study," Journal of the American College of Cardiology, vol. 31, no. 3, pp. 519-525, 1998.

[19] J. Danesh, R. Collins, and R. Peto, "Lipoprotein(a) and coronary heart disease: meta-analysis of prospective studies," Circulation, vol. 102, no. 10, pp. 1082-1085, 2000.

[20] Y. Momiyama, R. Ohmori, Z. A. Fayad et al., "Associations between serum lipoprotein(a) levels and the severity of coronary and aortic atherosclerosis," Atherosclerosis, vol. 222, no. 1, pp. 241-244, 2012.

[21] S. J. Nicholls, W. H. W. Tang, H. Scoffone et al., "Lipoprotein(a) levels and long-term cardiovascular risk in the contemporary era of statin therapy," Journal of Lipid Research, vol. 51, no. 10, pp. 3055-3061, 2010.

[22] S. S. Virani, A. Brautbar, B. C. Davis et al., "Associations between lipoprotein(a) levels and cardiovascular outcomes in black and white subjects: The Atherosclerosis Risk in Communities (ARIC) study," Circulation, vol. 125, no. 2, pp. 241-249, 2012.

[23] S. Tsimikas and J. L. Witztum, "The role of oxidized phospholipids in mediating lipoprotein(a) atherogenicity," Current Opinion in Lipidology, vol. 19, no. 4, pp. 369-377, 2008.

[24] J. Hoover-Plow and M. Huang, "Lipoprotein(a) metabolism: potential sites for therapeutic targets," Metabolism, vol. 62, no. 4, pp. 479-491, 2013.

[25] D. J. Grainger, P. R. Kemp, A. C. Liu, R. M. Lawn, and J. C. Metcalfe, "Activation of transforming growth factor- $\beta$ is inhibited in transgenic apolipoprotein(a) mice," Nature, vol. 370, no. 6489, pp. 460-462, 1994.

[26] F. Kronenberg, M. F. Kronenberg, S. Kiechl et al., "Role of lipoprotein(a) and apolipoprotein(a) phenotype in atherogenesis: Prospective results from the bruneck study," Circulation, vol. 100, no. 11, pp. 1154-1160, 1999.

[27] J. D. Spence and M. Koschinsky, "Mechanisms of lipoprotein(a) pathogenicity: prothrombotic, proatherosclerotic, or both," Arteriosclerosis, Thrombosis, and Vascular Biology, vol. 32, no. 7, pp. 1550-1551, 2012. 


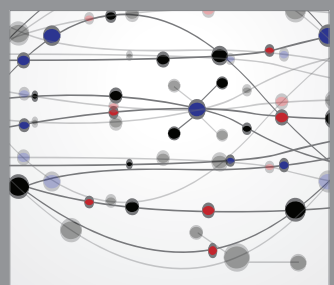

The Scientific World Journal
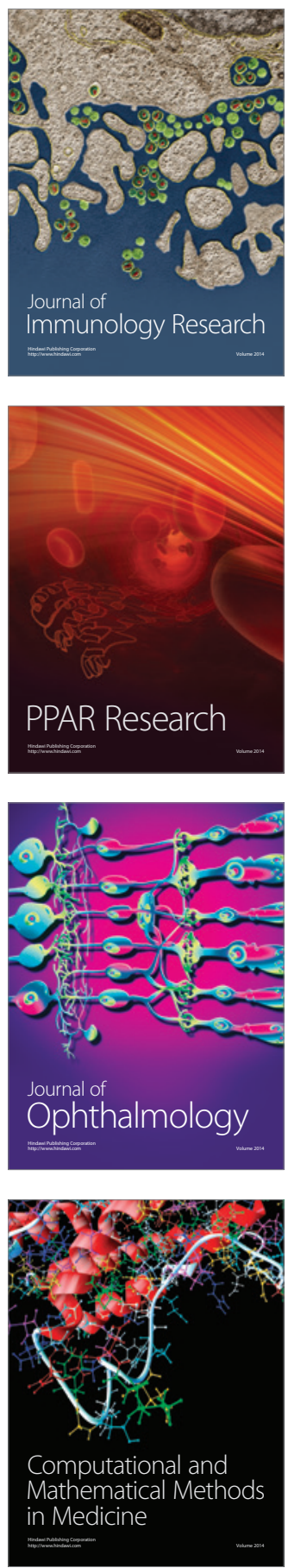

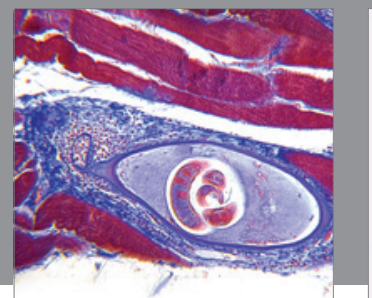

Gastroenterology

Research and Practice
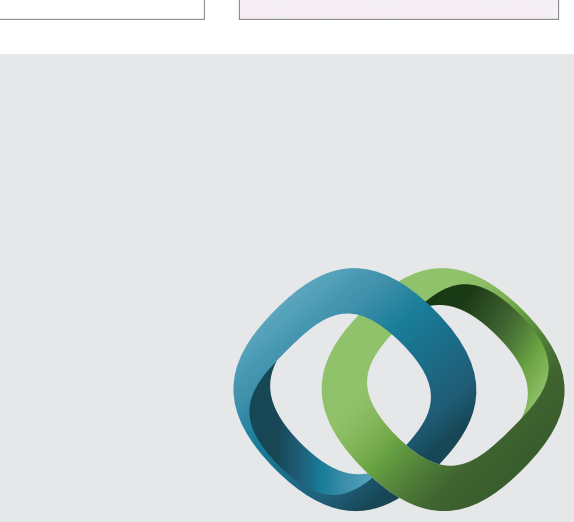

\section{Hindawi}

Submit your manuscripts at

http://www.hindawi.com
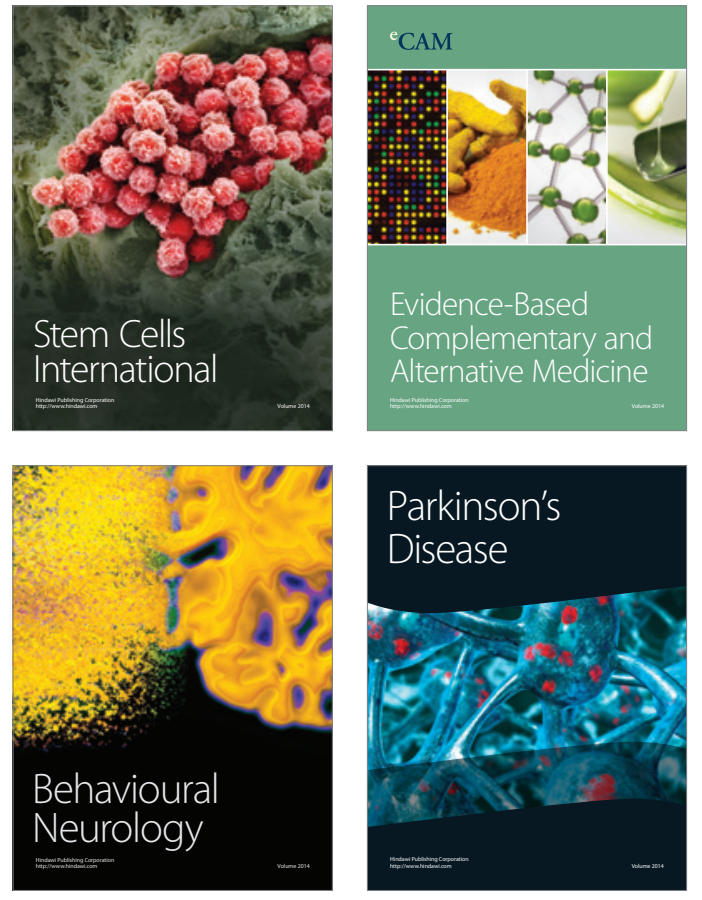
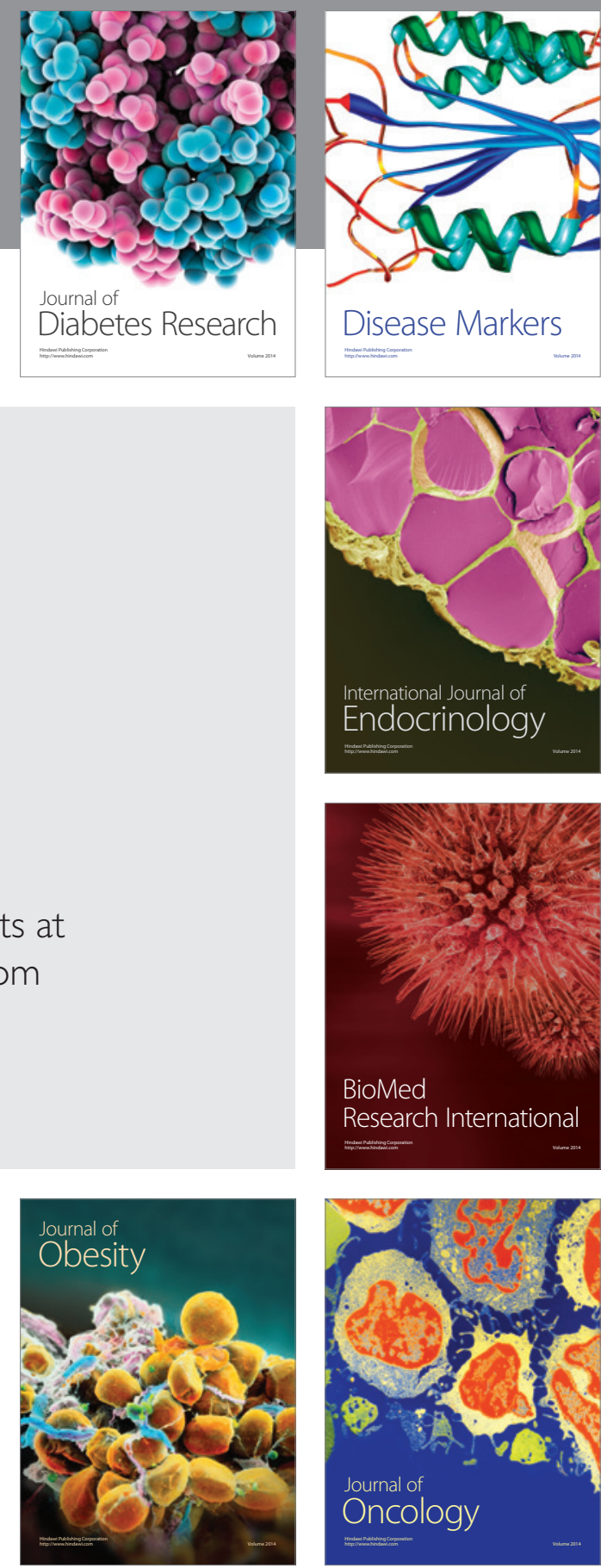

Disease Markers
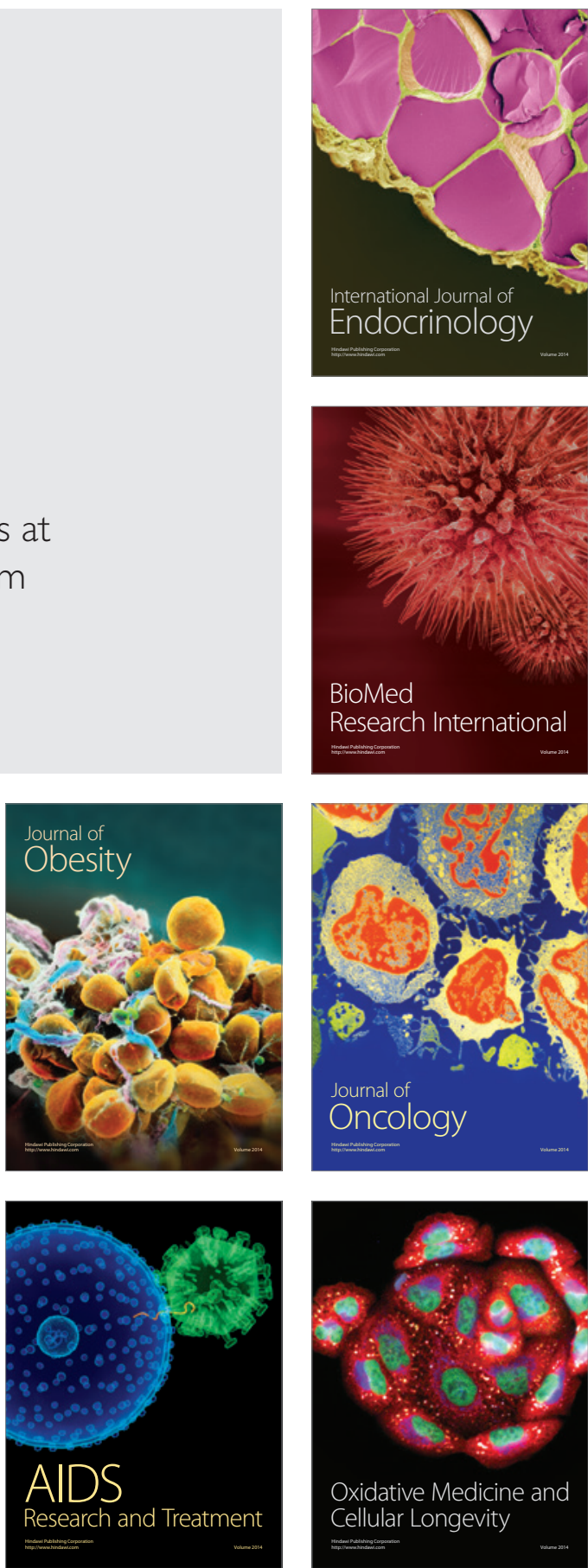